\title{
CONFORMALLY FLAT SPACES AND \\ A PINCHING PROBLEM \\ ON THE RICCI TENSOR
}

TH. HASANIS

ABSTRACT. Recent results of S. I. Goldberg on conformally flat manifolds and hypersurfaces of Euclidean space are extended.

1. Introduction. By applying S.-T. Yau's "maximum principle”, S. Goldberg [3] proved that an $n$-dimensional, $n \geqslant 3$, conformally flat Riemannian manifold with constant scalar curvature $R$ whose Ricci curvature is bounded below, and for which suptrace $Q^{2}<R^{2} /(n-1)$, is a space form. A corresponding result for hypersurfaces in Euclidean space was obtained by analogy.

The author sincerely thanks Professor S. Goldberg for valuable suggestions.

2. Preliminaries. Let $(M, g)$ be a Riemannian manifold with metric $g$. The curvature transformation $R(X, Y), X, Y \in T_{p} M$, where $T_{p} M$ is the tangent space at $p \in M$, and $g$ are related by

$$
R(X, Y)=\nabla_{[X, Y]}-\left[\nabla_{X}, \nabla_{Y}\right]
$$

where $\nabla_{X}$ is the operation of covariant differentiation with respect to $X$. In terms of a basis $X_{1}, \ldots, X_{n}$ of $T_{p} M$ we set

$$
R_{i j k l}=g\left(R\left(X_{i}, X_{j}\right) X_{k}, X_{l}\right), \quad R_{i j}=\operatorname{trace}\left(X_{k} \rightarrow R\left(X_{i}, X_{k}\right) X_{j}\right) .
$$

We denote the scalar curvature by $R$, that is, $R=$ trace $Q$, where $Q$ is the symmetric linear transformation field defined by the Ricci tensor, that is $Q=\left(R_{j}^{i}\right)$ and $R_{j}^{i}=g^{i k} R_{j k}$. The manifold $(M, g)$ is conformally flat if $g$ is conformally related to a locally flat metric. Let $M$ be an $n$-dimensional $(n \geqslant 3)$ conformally flat Riemannian manifold with constant scalar curvature, then the following formula may be found in [3]:

$$
\begin{aligned}
\frac{1}{2} \Delta \operatorname{trace} Q^{2}= & \frac{n}{n-2} \operatorname{trace} Q^{3}-\frac{2 n-1}{(n-1)(n-2)} R \text { trace } Q^{2} \\
& +\frac{R^{3}}{(n-1)(n-2)}+g(\nabla Q, \nabla Q) .
\end{aligned}
$$

Put $S=Q-R_{I} / n, I=$ identity. Then trace $S^{2} \geqslant 0$ with equality holding if and only if $M$ is an Einstein space. Obviously trace $S^{2}=\operatorname{trace} Q^{2}-R^{2} / n$, and since $R$

Received by the editors February 22, 1982.

1980 Mathematics Subject Classification. Primary 53C20; Secondary 53A07, 53C40.

Key words and phrases. Conformally flat, Ricci curvature, scalar curvature. 
is constant we get $\Delta$ trace $S^{2}=\Delta$ trace $Q^{2}$, where $\Delta$ is the Laplace operator on $M$. Repeating the same calculations as in [3] we get, for $f^{2}=\operatorname{trace} S^{2}$,

$$
\frac{1}{2} \Delta f^{2} \geqslant \sqrt{\frac{n}{n-1}} f^{2}\left(\frac{R}{\sqrt{n(n-1)}}-f\right) \text {. }
$$

The tool for the proof of the main result is a slight modification [5, Theorem 1] of the generalized maximum principle proved in [1 or 8], which we state as follows: Let $M$ be a complete, connected Riemannian manifold with Ricci curvature bounded from below. Let $f$ be a $C^{2}$-function bounded from above on $M$ and which has no maximum. Then for all $\varepsilon>0$, there exists a point $P \in M$ such that at $P$,

(1) $\sup f-\varepsilon<f(P)<\sup f-\varepsilon / 2$,

(2) $|\operatorname{grad} f|(P)<\varepsilon$,

(3) $\Delta f(P)<\varepsilon$.

3. Main results. The following lemma is fundamental and may be found in [6].

LEMMA. Let $a_{1}, \ldots, a_{n}$ be real numbers satisfying the inequality

$$
\sum_{i=1}^{n} a_{i}^{2}<\frac{1}{n-1}\left(\sum_{i=1}^{n} a_{i}\right)^{2}
$$

Then for any pair of distinct $i$ and $j=1, \ldots, n$ we have $a_{i} a_{j}>0$.

THEOREM 1. Let $M$ be an $n$-dimensional $(n \geqslant 3)$, complete, connected conformally flat Riemannian manifold. If its scalar curvature $R$ is a positive constant and trace $Q^{2} \leqslant R^{2} /(n-1)$, then $M$ is a space form or trace $Q^{2}=R^{2} /(n-1)$ everywhere on $M$.

Proof. Let $f^{2}$ be as in $\$ 2$ above; we distinguish two cases.

Case I. $f^{2}$ attains its maximum; then by using E. Hopf's well-known theorem we conclude from (2.1) that $f^{2}=$ constant and thus $f^{2}=0$ or $f^{2}=R^{2} / n(n-1)$ everywhere on $M$. But then trace $Q^{2}=R^{2} / n$, that is, $M$ is an Einstein space and thus a space form or trace $Q^{2}=R^{2} /(n-1)$ everywhere on $M$.

Case II. $f^{2}$ has no maximum. Suppose $\sup f^{2}<R^{2} / n(n-1)$; then from (2.1) and by using the same method as in the proof of $[4$, Theorem A] we conclude that $f^{2}=0$, that is, $M$ is a space form. Now let $\sup f^{2}=R^{2} / n(n-1)$. Since $f^{2}$ attains no maximum we also have $f^{2}<R^{2} / n(n-1)$. We prove that this is not true. Obviously, since $f^{2}<R^{2} / n(n-1)$, we get trace $Q^{2}<R^{2} /(n-1)$. Applying the lemma for the eigenvalues of the Ricci tensor we conclude that the Ricci curvature is bounded from below; in particular, it is positive. By generalized maximum principle we have that, for any natural number $m$, there exists a point $P_{m} \in M$ such that (since $\left.\sup f^{2}=R^{2} /(n(n-1))\right)$

$$
\begin{gathered}
\frac{R^{2}}{n(n-1)}-\frac{1}{m}<f^{2}\left(P_{m}\right)<\frac{R^{2}}{n(n-1)}-\frac{1}{2 m} \\
\sqrt{\frac{n}{n-1}} f^{2}\left(P_{m}\right)\left(\frac{R}{\sqrt{n(n-1)}}-f\left(P_{m}\right)\right) \leqslant \frac{1}{2} \Delta f^{2}\left(P_{m}\right)<\frac{1}{2 m} .
\end{gathered}
$$


From (3.1) we get

$$
\left(\frac{R}{\sqrt{n(n-1)}}-f\left(P_{m}\right)\right)\left(\frac{R}{\sqrt{n(n-1)}}+f\left(P_{m}\right)\right)>\frac{1}{2 m}
$$

or

$$
\frac{R}{\sqrt{n(n-1)}}-f\left(P_{m}\right)>\frac{1}{2 m\left(R / \sqrt{n(n-1)}+f\left(P_{m}\right)\right)}
$$

and thus (3.2) becomes

$$
\sqrt{\frac{n}{n-1}} f^{2}\left(P_{m}\right) \cdot \frac{1}{2 m\left(R / \sqrt{n(n-1)}+f\left(P_{m}\right)\right)}<\frac{1}{2 m}
$$

or

$$
\sqrt{\frac{n}{n-1}} f^{2}\left(P_{m}\right)<\frac{R}{\sqrt{n(n-1)}}+f\left(P_{m}\right)
$$

or

$$
f^{2}\left(P_{m}\right)-\sqrt{\frac{n-1}{n}} f\left(P_{m}\right)-\frac{R}{n}<0 .
$$

From (3.3), since $f\left(P_{m}\right)>0$, we get

$$
f\left(P_{m}\right)<\frac{\sqrt{n-1+4 R}+\sqrt{n-1}}{2 \sqrt{n}}
$$

and thus

$$
\sup f \leqslant \frac{\sqrt{n-1+4 R}+\sqrt{n-1}}{2 \sqrt{n}} .
$$

Now, sup $f=R / \sqrt{n(n-1)}$ and, comparing with the last inequality, we take

$$
\frac{R}{\sqrt{n(n-1)}} \leqslant \frac{\sqrt{n-1+4 R}+\sqrt{n-1}}{2 \sqrt{n}}
$$

or

$$
2 R-(n-1) \leqslant \sqrt{(n-1)^{2}+4 R(n-1)}
$$

or

$$
R \leqslant 2(n-1) .
$$

Now let $\lambda$ be a positive constant, then the Riemannian manifold $(M, \lambda g)$ has scalar curvature $\bar{R}=R / \lambda$ and satisfies the same assumptions as $(M, g)$. Then we must have, as above,

$$
\bar{R}=R / \lambda \leqslant 2(n-1) \text { or } R \leqslant 2 \lambda(n-1),
$$

which is impossible for $\lambda<R / 2(n-1)$. This completes the proof of the theorem. 
COROllary 1. Let $M$ be an $n$-dimensional $(n \geqslant 3)$, complete, connected conformally flat Riemannian manifold. If its scalar curvature $R$ is a positive constant and trace $Q^{2}<R^{2} /(n-1)$, then $M$ is a space form.

REMARK. If on a conformally flat Riemannian manifold with positive constant scalar curvature $R$, trace $Q^{2}=R^{2} /(n-1)$ everywhere, then it follows easily [2, Theorem 3] that $M$ is a Riemannian product of a space form $M_{1}$, with a 1-dimensional Riemannian manifold $N$, i.e., $M=M_{1} \times N$.

Thus we have

THEOREM $1^{\prime}$. The only $n$-dimensional $(n \geqslant 3)$, complete, connected conformally flat Riemannian manifolds with positive constant scalar curvature such that trace $Q^{2} \leqslant$ $R^{2} /(n-1)$, are the space forms and the Riemannian products $M_{1} \times N$ where $M_{1}$ is a space form and $N$ is 1-dimensional.

In a similar manner, we obtain the following extension of a theorem of Okumura [7].

THEOREM 2. Let $M$ be an $n$-dimensional $(n \geqslant 3)$, complete, connected hypersurface of Euclidean space $E^{n+1}$. If the mean curvature $H$ is constant and $S \leqslant n^{2} H^{2} /(n-1)$, where $S$ is the square of the second fundamental form, then $M$ is a hyperplane, $a$ hypersphere or a circular cylinder $S^{n-1} \times E$.

COROllary 2. Let $M$ be an n-dimensional $(n \geqslant 3)$, complete, connected hypersurface of $E^{n+1}$. If the mean curvature is constant and $S<n^{2} H^{2} /(n-1)$, then $M$ is a hypersphere.

\section{REFERENCES}

1. S. Y. Cheng and S.-T. Yau, Differential equations on Riemannian manifolds and their geometric applications, Comm. Pure Appl. Math. 28 (1975), 333-354.

2. S. I. Goldberg, On conformally flat spaces with definite Ricci curvature, Kōdai Math. Sem. Rep. 21 (1969), 226-232.

3. An application of Yau's maximum principle to conformally flat spaces, Proc. Amer. Math. Soc. 79 (1980), 268-270.

4. Th. Hasanis, Characterization of totally umbilical hypersurfaces, Proc. Amer. Math. Soc. 81 (1981), 447-450.

5. K. Motomiya, On functions which satisfy some differential inequalities on Riemannian manifolds, Nagoya Math. J. 81 (1981), 57-72.

6. M. Okumura, Submanifolds and a pinching problem on the second fundamental tensors, Trans. Amer. Math. Soc. 178 (1973), 285-291.

7. Hypersurfaces and a pinching problem on the second fundamental tensor, Amer. J. Math. 86 (1974), 207-213.

8. S.-T. Yau, Harmonic functions on complete Riemannian manifolds, Comm. Pure Appl. Math. 28 (1975), 201-228.

Department of Mathematics, University of IoANnina, IoANnina, Greece 